\title{
The state of the focus and image quality of the Spitzer Space Telescope as measured in orbit
}

Robert Douglas Gehrz, Edward A. Romana, William F. Hoffmann, John P. Schwenker, John Eric Mentzell, et al.

Robert Douglas Gehrz, Edward A. Romana, William F. Hoffmann, John P. Schwenker, John Eric Mentzell, Joseph L. Hora, Peter R. Eisenhardt, Bernhard Rainer Brandl, Lee Armus, Karl R. Stapelfeldt, Dean C. Hines, Amanda K. Mainzer, Erick T. Young, David G. Elliott, "The state of the focus and image quality of the Spitzer Space Telescope as measured in orbit," Proc. SPIE 5487, Optical, Infrared, and Millimeter Space Telescopes, (12 October 2004); doi: 10.1117/12.551930

Event: SPIE Astronomical Telescopes + Instrumentation, 2004, Glasgow, United Kingdom 


\title{
The state of the focus and image quality of the Spitzer Space Telescope as measured in orbit
}

\author{
Robert D. Gehrz ${ }^{*}$, Edward A. Romana ${ }^{* *}$, William F. Hoffmann ${ }^{\mathrm{c}}$, John P. Schwenker ${ }^{\mathrm{d}}$, John E. \\ Mentzell $^{\mathrm{e}}$, Joseph L. Hora ${ }^{\mathrm{f}}$, Peter R. Eisenhardt ${ }^{\mathrm{b}}$, Bernhard R. Brandl ${ }^{\mathrm{g}}$, Lee Armus ${ }^{\mathrm{h}}$, Karl R. \\ Stapelfeldt ${ }^{\mathrm{b}}$, Dean C. Hines ${ }^{\mathrm{c}}$, Amanda K. Mainzer ${ }^{\mathrm{b}}$, Erik T. Young ${ }^{\mathrm{c}}$, and David G. Elliott ${ }^{\mathrm{b}}$ \\ a Department of Astronomy, University of Minnesota; ${ }^{b} J P L$ Caltech, 'Steward Observatory, \\ University of Arizona, ${ }^{\mathrm{d} B a l l}$ Aerospace Technologies Corporation, ${ }^{\mathrm{e}}$ NASA Goddard Space Flight \\ Center, ${ }^{f}$ Harvard-Smithsonian Center for Astrophysics, ${ }^{g}$ Sterrewacht Leiden/Cornell University, \\ ${ }^{\mathrm{h}}$ Spitzer Science Center, Caltech
}

\begin{abstract}
We describe the process by which the NASA Spitzer Space Telescope (SST) Cryogenic Telescope Assembly (CTA) was brought into focus after arrival of the spacecraft in orbit. The ground rules of the mission did not allow us to make a conventional focus sweep. A strategy was developed to determine the focus position through a program of passive imaging during the observatory cool-down time period. A number of analytical diagnostic tools were developed to facilitate evaluation of the state of the CTA focus. Initially, these tools were used to establish the in-orbit focus position. These tools were then used to evaluate the effects of an initial small exploratory move that verified the health and calibration of the secondary mirror focus mechanism. A second large move of the secondary mirror was then commanded to bring the telescope into focus. We present images that show the CTA Point Spread Function (PSF) at different channel wavelengths and demonstrate that the telescope achieved diffraction limited performance at a wavelength of $5.5 \mu \mathrm{m}$, somewhat better than the level-one requirement.
\end{abstract}

Keywords: Spitzer Space Telescope (SST), SIRTF, IRAC, IRS, MIPS, noise-pixels, cryogenic, telescope, focus

\section{INTRODUCTION}

NASA's Spitzer Space Telescope (SST)***, launched on 25 August, 2003, is an infrared (IR) observatory that is being used to study the creation of the universe, the formation and evolution of primitive galaxies, the genesis of stars and planets, and the chemical evolution of the universe. Spitzer's $85-\mathrm{cm}$ cryogenically cooled beryllium Ritchey-Chretien telescope operates at temperatures as low as $5.5 \mathrm{~K}$. The Cryogenic Telescope Assembly (CTA) houses the cryogenic telescope, the liquid helium dewar, and the science instruments. Fulfillment of the Spitzer science objectives requires that the focal plane images delivered by the CTA meet a Level-One-Requirement (LOR) of diffraction limited (DL) performance at a wavelength of $6.5 \mu \mathrm{m}$. A telescope that is DL at $6.5 \mu \mathrm{m}$ is defined to have an optical wave-front error of $\lambda / 14(0.464 \mu \mathrm{m}$ RMS $)$ and produces a star image core whose peak energy is reduced by no more than $80 \%$ compared to the ideal telescope (Strehl ratio of 0.8$)^{1,2}$. During In-Orbit Check-Out (IOC), the CTA was focused by moving the secondary mirror using a cryogenic focus mechanism. Because a failure of the secondary mirror focus mechanism could have caused a single-point failure in the Spitzer mission's ability to meet its science objectives, it was desirable to bring the CTA into focus with a minimal number of focus motor actuations rather than performing a conventional focus sweep. Using a number of analysis tools developed by Hoffmann et al. $^{3}$ and tested by the Spitzer IOC Focus Integrated Products Team (IPT) prior to launch as discussed by Gehrz and Romana ${ }^{4}$, we determined the magnitude and direction of the secondary mirror move necessary to focus the telescope by passively imaging stars during the CTA in-orbit cool-down period. In this paper, we describe the process by which the Spitzer CTA was brought into focus by evaluating the shapes of star images made with the Infrared Array Camera (IRAC) ${ }^{5}$ and the other

* gehrz@astro.umn.edu; phone 612-624-7806; fax: 612-626-2029; Department of Astronomy, School of Physics and Astronomy, 116 Church Street, S.E., University of Minnesota, Minneapolis , MN, USA, 55455; ** eromana@Spitzerweb.jpl.nasa.gov; phone 818- 3541106; fax 818-393-6236, Jet Propulsion Laboratory, MS 264-767, 4800 Oak Grove Drive, Pasadena, CA, USA, 91109 ; *** formerly Space Infrared Telescope Facility (SIRTF). 
Science Instruments (SI's). We show how these images were used to predict the direction and magnitude of the secondary mirror motion required to reach best focus. We describe the results of a small initial exploratory move that was used to verify the functional status of the secondary mirror focus mechanism and those of a second larger move of the secondary mirror that was commanded to bring the telescope into focus. We present images that show that the achieved CTA point spread function (PSF) is diffraction limited at a wavelength of $5.5 \mu \mathrm{m}$, somewhat better than the level one requirement.

\section{FOCUS SUCCESS CRITERIA AND REFOCUS STRATEGY}

The Spitzer Science Working Group (SWG) determined during its pre-launch deliberations that the mission's level-one science objectives would be met if no imaging channel for any SI would be degraded in integration time by more than $10 \%$ averaged over the focal plane with respect to the best focus for that channel. Analysis conducted by the Focus IPT showed that this condition is met for all SI channels when the secondary mirror is within $+/-3 \mu \mathrm{m}(0.3 \mathrm{~mm}$ at the telescope F/12 focus ) of the IRAC best compromise focus position. The IPT adopted a refocus strategy that would minimize the number of secondary mirror focus motor actuations. During the CTA cool-down we conducted fourteen passive imaging campaigns to evaluate the state of the CTA image quality and focus position as the telescope and its metering structure reached thermal equilibrium. On day 38 after launch, the CTA secondary mirror was determined to be too far from the primary mirror by 17 $\mu \mathrm{m}$ with respect to the desired best compromise focus position. Correction of the focus therefore dictated that the secondary mirror be moved $17 \mu \mathrm{m}$ closer to the primary mirror. A $2 \mu \mathrm{m}$ secondary mirror move in this direction was commanded. Evaluation of stellar images after this move confirmed that the mirror had moved by the predicted amount in the correct direction. A $13 \mu \mathrm{m}$ move was then commanded to move focus to the edge of the acceptable focus tolerance band without overshooting. Quantitative evaluation of the images following this second move showed that the LOR for image quality had been met and plans to conduct a final small third focus move were cancelled. In the sections that follow, we describe the focus evaluation, the refocus process, and image quality in quantitative terms.

\section{TRACKING THE CTA FOCUS DURING IN-ORBIT COOL-DOWN}

Figure 1 presents the CTA predicted and observed focus positions as a function of time. CTA cool-down temperatures are indicated for three data gathering campaigns (E, J, and Q). On the basis of results of extensive ground-based testing, the CTA focus (the spacing between the secondary and primary mirrors) was set to the predicted best cold focus position. The predicted focus position cooling trend shown in Figure 1 was determined by applying an optical and thermal model

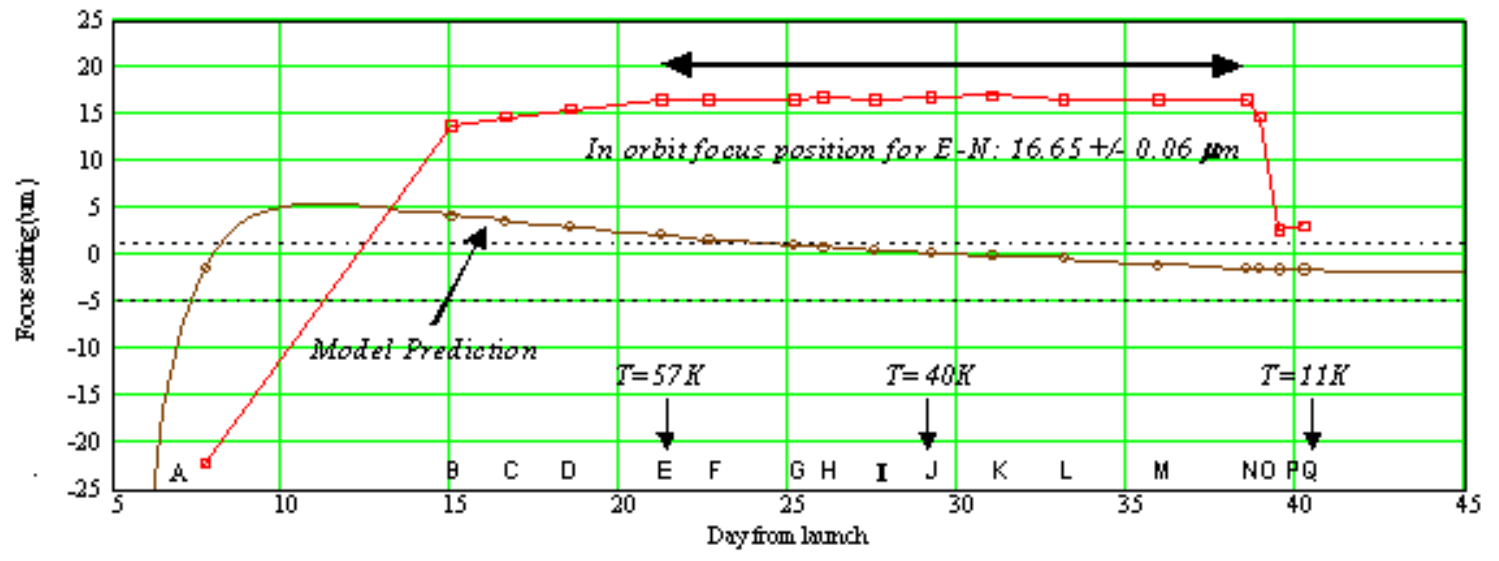

Figure 1. Secondary mirror position versus day from launch. The dashed lines designate the boundaries of the acceptable focus position tolerance band of $+/-3 \mu \mathrm{m}$ from the target best compromise focus position of $-1.8 \mu \mathrm{m}$ reckoned with respect to our historical hardware and optical model fiducial. Open circles $(O)$ are the predicted IRAC focus position trend calculated with a model that links individual optical, mechanical and thermal models. Square boxes ( $\square$ ) are the IRAC in-orbit focus determination results computed by the Simfit method (Hoffmann et al. ${ }^{10}$ ). The discrepancy between the actual and model focus positions is most likely due to uncertainties in values for low temperature material properties of the CTA. Telescope focus moves were commanded during the spacecraft uplink and downlink communication events designated with the letters $\mathrm{O}$ and $\mathrm{P}$. A third planned focus move was not executed because the image quality requirements stated in the LOR were met after the second focus move. 
of the CTA (Schwenker et al. ${ }^{6}$ ). Following the launch of the warm telescope, cool-down focus position data are sparse until day 15 . Between days 8 and 15 , the data show that the secondary mirror position changed from $-22 \mu \mathrm{m}$ to $+14 \mu \mathrm{m}$. Following this early time period, focus position moved more slowly from $+14 \mu \mathrm{m}$ to $+17 \mu \mathrm{m}$. From day 21 when the CTA temperature was $57 \mathrm{~K}$ through day 38 when the CTA temperature was $11 \mathrm{~K}$, the focus position remained stable at $+17 \mu \mathrm{m}$,. The two secondary mirror moves, conducted during days 38 and 39 , brought the focus position to $+3.0 \mu \mathrm{m}$ where the quantitative analysis described in section 6 revealed that the telescope met the performance requirement set in the LOR.

\section{SCIENCE INSTRUMENT CONFOCALITY AS MEASURED IN-ORBIT}

The Spitzer Confocality Schematic Diagram (Figure 2) shows the best focus position for the CTA instrument channels. The indicated focus positions have been determined at different milestone points during the project's pre-launch testing program and during IOC. The scatter in the focus positions of the fourteen Science Instrument (SI) channels requires that the CTA focus be set to a "best compromise focus" position that is consistent with the success criteria described inSection 2 above. The confocality departures seen in Figure 2 arise from a combination of measurement, testing, and

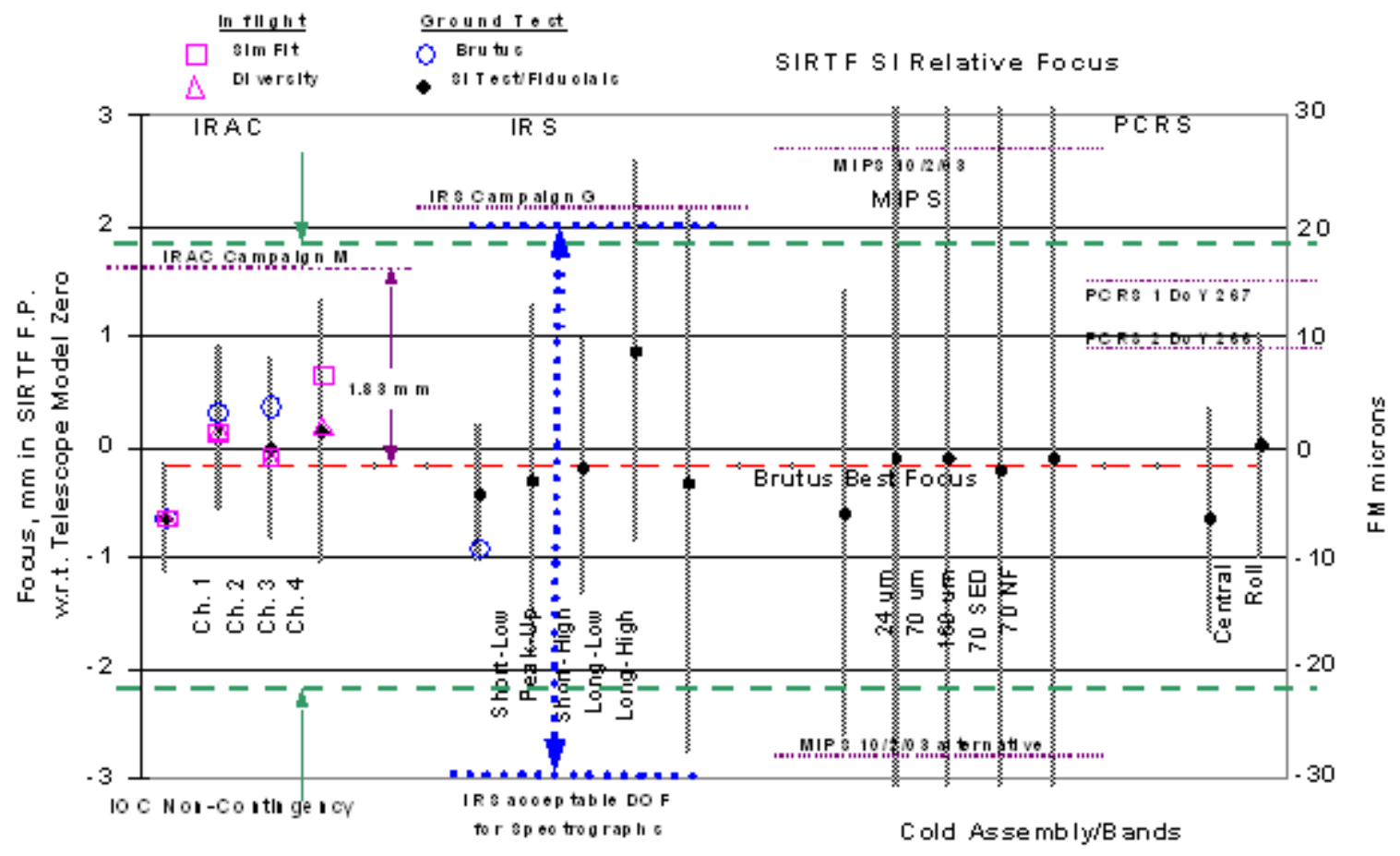

Figure 2. The Spitzer SI channel confocality schematic diagram. The left vertical axis is in mm at the F/12 focus. The right vertical axis is secondary mirror displacement in $\mu \mathrm{m}$. The black dots $(\bullet)$ represent the prelaunch measured focus positions of the SI focal planes. These measurements were determined by optical cathetometer measurements of channel fiducials performed during the integration of each instrument into the CTA multiple instrument chamber (MIC). The gray vertical bars represent the acceptable depth of focus tolerance band (Strehl of 0.90) for each instrument channel. The open circles $(\bigcirc)$ represent measured pre-launch, cryogenic temperature, end to end autocollimation channel focus positions. The open square boxes $(\square)$ are actual in-orbit focus position determination results obtained by the simfit method (Hoffmann et al. ${ }^{10}$ ). The focus zero point is defined with respect to an historical optical model origin. The optical model of the telescope and its instruments is assembled from weighted interferometric testing results gathered at numerous warm and cold integration milestones during component, sub assembly, assembly, and the end-to-end BRUTUS auto-collimation test with the OSCAR cryogenic reference flat (Schwenker et al. ${ }^{6,7}$ ). 


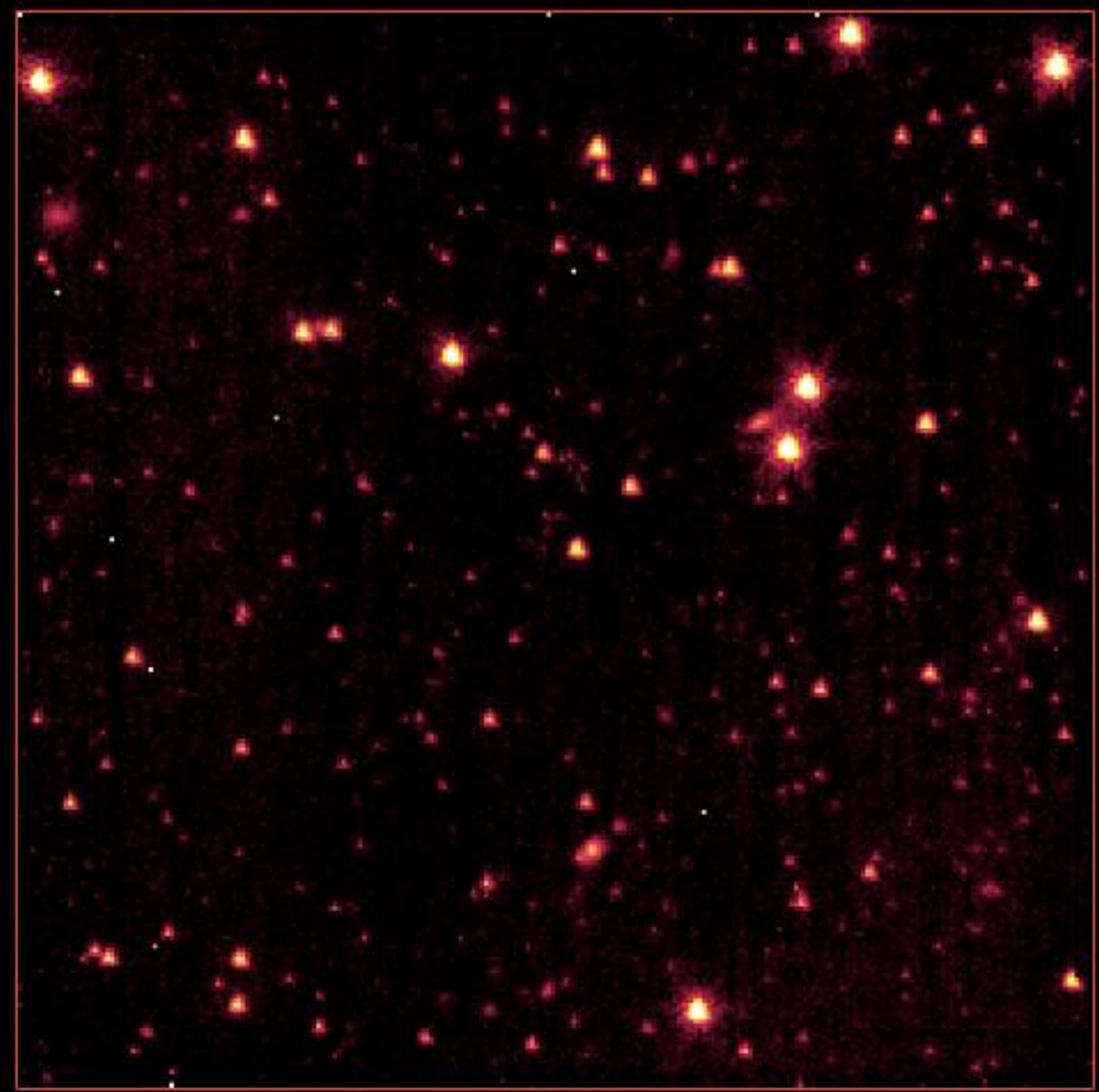

SIRTF "Aliveness Test" Image

Figure 3. The first Spitzer IRAC channel $1(3.6 \mu \mathrm{m})$ "aliveness test" image. This image shows that the CTA arrived in orbit only slightly out of focus and that for saturated bright stars, the core has a triangularly shaped skirt. This "trefoil" aberration is due to a secondary mirror edge figure error introduced by the secondary mirror support system. As the CTA focus was improved, this trefoil term was diminished (see Figure 4). Because the trefoil term was not large enough to degrade the LOR of diffraction limited performance at $6.5 \mu \mathrm{m}$, the SST Project elected to conserve cost and schedule margin by leaving the trefoil term uncorrected. 
construction limitations in each channel at its operating temperature and wavelength. Other measurements during MIC and CTA integration accrue additional confocality errors. Because the acceptable focus tolerance band scales linearly with wavelength ${ }^{1}$, confocality is more important for the CTA short wavelength channels. Based upon the results of the end-to end ground based SIRTF Optical Performance Test (the BRUTUS Test ${ }^{6,7}$ ), the spacing between the secondary and primary mirrors was set to the expected in-orbit best compromise focus position. This position is shown by the horizontal dashed red line. During the BRUTUS optical testing, due to some uncertainties concerning precise knowledge of the radius of curvature (power) of the cryogenic auto-collimation flat $\left(\mathrm{OSCAR}^{6,7}\right)$, the $3 \sigma$ uncertainty in the focus setting was a comparatively large $\pm 2 \mu \mathrm{m}$ secondary mirror position ( $\pm 2.0 \mathrm{~mm}$ at the telescope $\mathrm{F} / 12$ focus $)$. This tolerance band is shown by the horizontal dashed green lines. Thus, there was a reasonable expectation that Spitzer would arrive in orbit at a focus position that would compromise the capabilities of the short IR wavelength instrument channels. Therefore an in orbit refocus activity was incorporated into the IOC time-line prior to launch. Once in orbit, we found images that revealed a focus position and channel parfocality consistent with our expectations as a result of the focus setting and verification activities conducted during the IRAC, $\operatorname{IRS}^{8}$, and MIPS ${ }^{9}$ BRUTUS test programs (Figure 3).

\section{SCIENCE INSTRUMENT IMAGES OBTAINED DURING IN-ORBIT CHECKOUT}

The initial "aliveness test" image obtained with IRAC Channel $1(3.6 \mu \mathrm{m})$, shown in Figure 3, verified that the telescope had arrived in orbit with the focus point $2.3 \mathrm{~mm}$ below the MIC focal plane. This image verified that the sign and magnitude of the OSCAR power had been accounted for correctly in setting the launch focus position ${ }^{6}$. Had a sign error occurred, the focal point of the CTA would have arrived in orbit too far from the focal plane for the secondary mirror focus mechanism movement range to bring the CTA into focus. Images made with the IRAC $3.6 \mu \mathrm{m}$ array (Figure 4 ), the IRS $16.1 \mu \mathrm{m}$ blue peakup array (Figure 5), and the MIPS $24 \mu \mathrm{m}$ array (Figure 6) show CTA performance before and after the refocus program. There was Strehl ratio gain of a factor of 1.8 in IRAC channel 1. There were less dramatic gains for channels 2 ( $4.5 \mu \mathrm{m}$; factor of 1.3) and $3(5.8 \mu \mathrm{m}$; factor of 1.17) as expected. There was no gain for channel $4(8.0 \mu \mathrm{m}$, factor of 1.0$)$. We interpret these results as being consistent with CTA diffraction limited performance at $5.5 \mu \mathrm{m}$. As expected from the IRAC results, negligible improvement was seen in the IRS short wavelength spectrometer slits, the IRS blue peak-up array, and the MIPS $24 \mu \mathrm{m}$ images after refocus. These conclusions are summarized in Sections 6 and 7 of this paper.
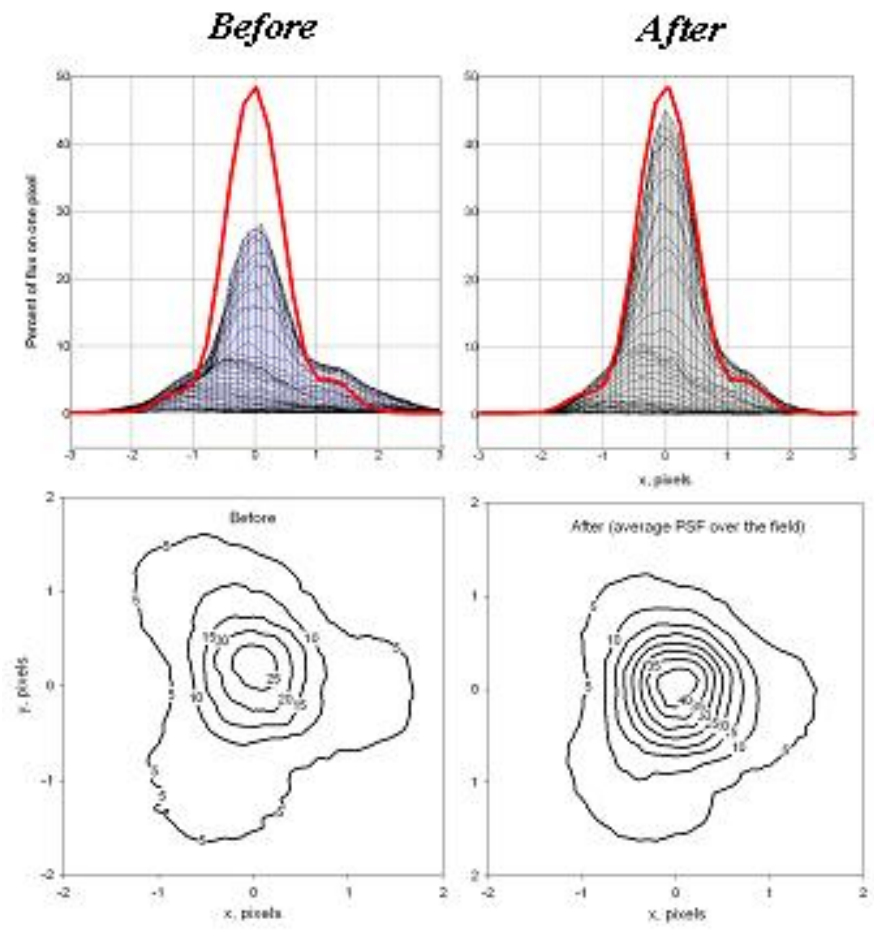

Figure 4. Top panels: The solid curves are a cross section of the pre-launch optical model point spread function (PSF) for best focus at the center of the IRAC channel 1 field. The solid surface is the actual in-flight PSF averaged over the field of view. The vertical axis is in units of percent of total flux collected by one pixel. The horizontal axis is in units of pixels. Bottom panels: The isophotal contours are in units of total flux collected on one pixel centered at the field coordinates indicated on the $\mathrm{x}$ and $\mathrm{y}$ axes. The relative amount of energy contained in the trefoil skirt was reduced after refocusing the CTA. 

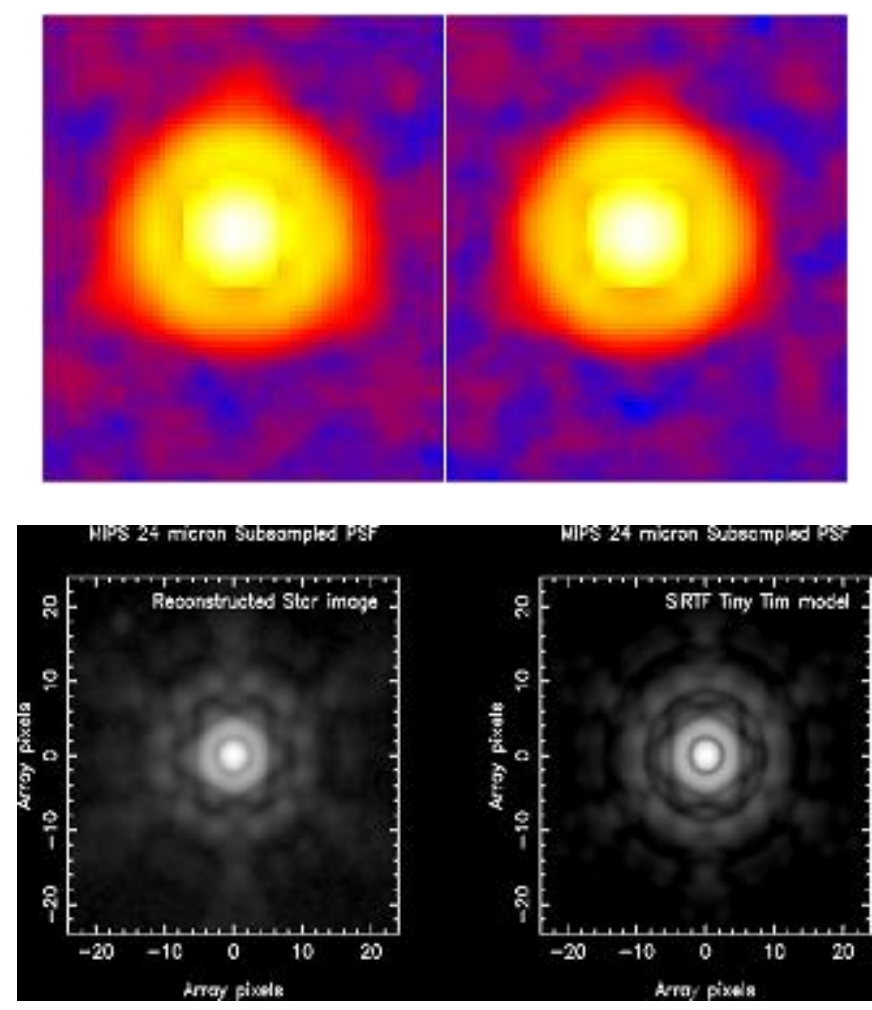

Figure 5. IRS Blue Peak-up Array $(16.1 \mu \mathrm{m})$ image before (left) and after (right) the IOC focus campaign. No substantial difference is evident

\section{DETERMINING FOCUS AND IMAGE QUALITY USING NOISE-PIXELS}

A small point source image core is always desirable. A small core increases the number of image information pixel elements and the power deposited on each pixel increases as the inverse square of the image core diameter. However, even for the ideal telescope at prime focus, the maximum achievable image sharpness is governed by the laws of physical diffraction that define a minimum achievable image core. For any ideal instrument of circular entrance aperture diameter D observing at a wavelength $\lambda$, the image of a true point source is a ringed diffraction pattern with a central maximum or core. This central core has an angular half width at zero intensity of $\theta=1.22 \lambda / \mathrm{D}$ (the Rayleigh Criterion). This phenomenon is explained by Huygens, who introduced the construction that every point of the optical wave-front surface gives rise to a propagating secondary spherical optical disturbance or wavelet, and Fresnel who furthered this construction with the principle of wavelet interference for all image points ${ }^{1}$. When the diffraction image is examined with an array detector having pixels that are sufficiently small so that the image core is well sampled, the number of pixels with an appreciable signal count is minimized and the mean photon count in these pixels is maximized (i.e. the image is small and bright). If we regard reflecting telescopes as achromatic instruments, in practice there always exists a wavelength below which a point source image core fails to obey the Rayleigh Criterion angular half width formula, and the core diameter remains constant. This is the diffraction limited wavelength (DLW) that characterizes the quality of the telescope as manufactured. At the DLW, the height variation of the optical surfaces in the manufactured telescope is such that at the focus point rays of a geometric ray trace will have traversed optical path lengths whose distances differ in a statistically significant manner by a fraction of a wavelength which prevents constructive Fresnel interference of Huygens wavelets. ${ }^{1}$. This decoherence moves energy from the central core to a neighborhood surrounding the core and degrades the Strehl ratio. The DLW marks the transition between physical and geometrical optics. Defocus will degrade and lengthen the achieved telescope DLW. The Spitzer DLW is specified to be $6.5 \mu \mathrm{m}$. The Spitzer DLW is above IRAC channels 1 and 2, and is in the channel 3 passband of IRAC. This decision was made to minimize telescope manufacturing costs. All the SI instrument channels were constructed to be DL at their operating wavelengths. Because the minimum image size diameter is limited by diffraction or geometry, there is an acceptable focus tolerance band or depth of focus within which performance is independent of focus position. Nevertheless, because of the non-confocality of IRAC channels 1 and 2, small telescope focus moves then benefitted one channel at the expense of the other. In the case of Spitzer, this led to a requirement for a focus position tolerance that was narrower than the acceptable focus position range of any given SI channel. We will quantify the notion of the area of a point source image by defining the 
equivalent number of pixels occupied by the image on a pixelated detector according to the Noise-pixels (NP) statistic.

6.1 Mathematical definition of the Noise-pixels (NP) statistic

Noise-pixels (NP) are the ratio of the sum squared over the sum of squares of the signal counts in $N$ pixels or bins:

$$
N P=\frac{\left(\sum_{j=1}^{N} p(j)\right)^{2}}{\sum_{j=1}^{N} p(j)^{2}}
$$

where $p(j)$ is the number of detected counts in the $\mathrm{j}^{\text {th }}$ pixel, and $\mathrm{N}$ is the number of pixels over which NP is calculated. The cardinal properties of NP are these:

1) NP is a measure of the spatial energy dispersion calculated over N pixels. Although NP is dimension-less, NP is a measure of the solid angle spanned by the image expressed in units of the detector pixels.

2) An image with all the energy in one pixel has NP $=1$

3) A uniform PSF with all the energy in $\mathrm{N}$ pixels has $\mathrm{NP}=\mathrm{N}$

4) Integration time on a point source scales linearly with NP in the photon noise regime. The same is true for Background Limited Incident Power (BLIP) observations.

\begin{tabular}{|c|c|c|c|c|c|}
\hline Campaign & Chan 1 & Chan 2 & Chan 3 & Chan 4 & Comm ent, Telescope T emperature \\
\hline A & 11.1 & 14.4 & & & $128 \mathrm{~K}$ \\
\hline B & 12.0 & 8.2 & 10.9 & 13.7 & $76 \mathrm{~K}$ \\
\hline C & 12.7 & 9.1 & 12.6 & 13.8 & $70 \mathrm{~K}$ \\
\hline D & 13.3 & 9.4 & 13.0 & 13.9 & $64 \mathrm{~K}$ \\
\hline E & 13.9 & 9.5 & 13.1 & 13.9 & CTA focus stabilizes at 57K \\
\hline F & 13.9 & 9.8 & 13.3 & 13.9 & $54 \mathrm{~K}$ \\
\hline G & 14.1 & 9.7 & 12.9 & 13.9 & $48 \mathrm{~K}$ \\
\hline H & 14.1 & 10.0 & 13.5 & 13.9 & $46 \mathrm{~K}$ \\
\hline I & 14.2 & 9.7 & 13.3 & 13.9 & $43 \mathrm{~K}$ \\
\hline J & 14.0 & 9.7 & 12.7 & 13.8 & $40 \mathrm{~K}$ \\
\hline K & 14.2 & 9.5 & 13.4 & 13.9 & $37 \mathrm{~K}$ \\
\hline L & 14.1 & 9.8 & 13.2 & 13.8 & $33 \mathrm{~K}$ \\
\hline M & 13.9 & 9.9 & 12.5 & 13.9 & $26 \mathrm{~K}$ \\
\hline N & 14.1 & 9.8 & 12.9 & 13.8 & Last pre-fo cus m easurem ent, $19 \mathrm{~K}$ \\
\hline O & 12.5 & 9.0 & 12.5 & 13.8 & After a $-2.01 \mu \mathrm{m}$ m ove, $17 \mathrm{~K}$ \\
\hline P & 7.7 & 7.6 & 11.1 & 14.0 & After a $-12.395 \mu$ m move, $15 \mathrm{~K}$ \\
\hline Q & 7.7 & 7.6 & 11.2 & 13.9 & Confirm ation m ea sure ment, $11 \mathrm{~K}$ \\
\hline
\end{tabular}

Table 1. Columns 2-5 give the mean Noise-pixels (NP) obtained during the in orbit checkout (IOC) data gathering campaigns for IRAC channels 1 through 4 . The NP values were calculated in a $13 \times 13$ pixel box which includes most the point source flux. Campaign P NP values of 7.7 and 7.6 are well within prelaunch requirement and expectation. There were no substantial focus changes during IRAC Campaigns E-N. 


\subsection{Noise-Pixel determination of the telescope best focus position}

The NP focus position determination method assumes that a quadratic relationship exists between NP and the separation of the primary and secondary mirrors of the CTA as described by Hoffmann et al. ${ }^{3}$. Best focus is the minimum of the focus characteristic equation. NP data from 3 telescope focus positions were used to solve for the 3 coefficients of the focus quadratic. After careful consideration of the parfocality diagram (Figure 2), the CTA telescope best focus position was defined as the best focus average of all images for the IRAC channels 1 and 2. This focus position met the focus success criteria for all of the other SI channels. Best focus was determined from the NP data gathered with the telescope in thermal steady state at $11 \mathrm{~K}$ at 3 different focus positions during 4 separate IOC data gathering campaigns. These focus campaigns were:

1) Campaign N: Determination of the initial in-orbit focus position

2) Campaign O: Determination of the focus position following a small exploratory move

3) Campaign P: Determination of the focus position following a large move toward best focus

4) Campaign Q: Confirmation that the best compromise focus had been achieved

Each campaign consisted of data from 25 field locations on a 5 by 5 array that spanned the detector field. At each field location, data gathering was ordinarily repeated 4 times with small dithered pointing offsets (of order a pixel fraction) designed to mitigate flat fielding and pixel phase effects. Campaigns I, M, and Q were executed with 12 dithers. Campaigns A-N each contained a total of 100 images. Campaign Q contained 300 images. The focus data analysis results are summarized in the tables 1-3. Figures 8 and 9 present the NP dispersion for IRAC channels 1 and 2 obtained in focus campaigns $\mathrm{N}$ and $\mathrm{P}$. The dispersion about the mean is caused by a number of effects including pixel phase, field curvature, focal plane tilt, flat fielding effects, and optical distortion.

\begin{tabular}{|c|c|c|r|c|}
\hline Campaign & $\begin{array}{c}\text { Focus } \\
\text { position }(\mu \mathrm{m})\end{array}$ & $\begin{array}{c}\text { IRAC } \\
\text { Channel }\end{array}$ & $\begin{array}{c}\text { Channel } \\
\text { Mean NP }\end{array}$ & Channel NP Variance \\
\hline $\mathrm{N}$ & $0.0(\mathrm{ref})$ & 1 & 13.1492 & 1.92157 \\
\hline $\mathrm{O}$ & -2 & 1 & 11.7725 & 2.27222 \\
\hline $\mathrm{P}$ & -14.4 & 1 & 7.0104 & 1.62675 \\
\hline $\mathrm{N}$ & $0.0(\mathrm{ref})$ & 2 & 9.21474 & 2.14453 \\
\hline $\mathrm{O}$ & -2 & 2 & 8.55388 & 1.89783 \\
\hline $\mathrm{P}$ & -14.4 & 2 & 7.21095 & 1.63191 \\
\hline
\end{tabular}

Table 2. Noise-pixels values and Focus Position for IRAC Channels 1 and 2 for campaigns N, O, and P

\begin{tabular}{|c|c|c|c|}
\hline Characteristic Focus Equations & $\begin{array}{c}\text { Best Focus } \\
\text { Position }(\mu \mathrm{m})\end{array}$ & $\begin{array}{c}\text { NP at Best } \\
\text { Focus }\end{array}$ & IRAC Channel \\
\hline $0.0215 * x^{\wedge} 2+0.736 * x+13.149$ & -17.0852 & 6.85492 & 1 \\
\hline $0.0154 * \mathrm{x}^{\wedge} 2+0.361 * \mathrm{x}+9.2147$ & -11.711 & 7.09941 & 2 \\
\hline Average & -14.3981 & 6.97715 & \\
\hline
\end{tabular}

Table 3. IRAC Channels 1 and 2 Focus Characteristic Equations. The minimum of each equation is best focus for that channel. The CTA telescope best focus is defined to be the average of the IRAC channel 1 and 2 best focus. 

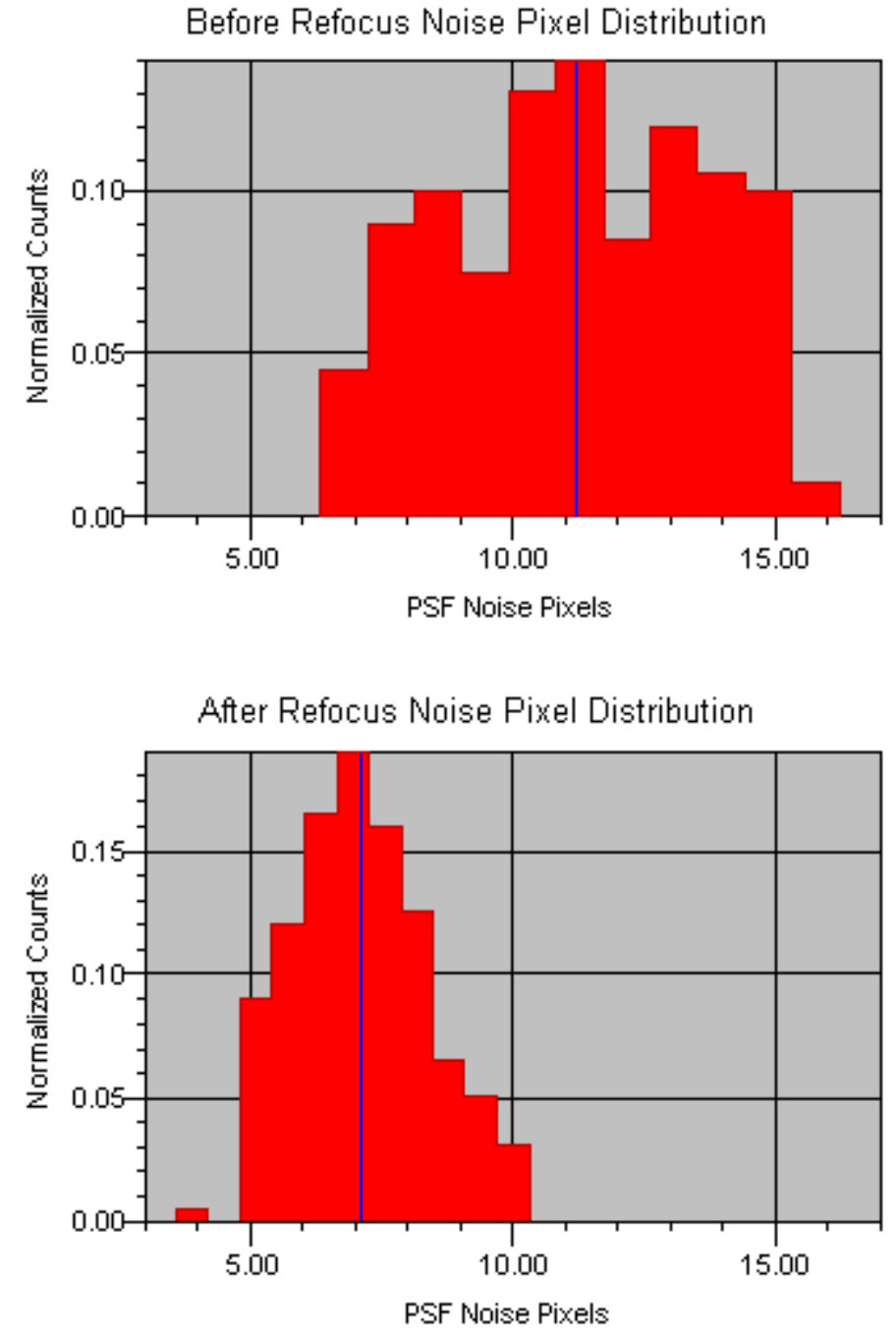

Figure 7. Noise-Pixel distribution for IRAC channels 1 and 2 after refocus: Mean $=11.181 \mathrm{NP}$, Variance $=5.903$ $\mathrm{NP} N=200$.
Figure 8. Noise-Pixel distribution for IRAC channels 1 and 2 after refocus. Mean $=7.111 \mathrm{NP}$, Variance $=1.63 \mathrm{NP}$ $\mathrm{N}=200$

\section{THE OPTICAL PERFORMANCE OF SPITZER}

7.1 Determination of the Diffraction Limited Wavelength of a telescope with an under sampled detector. Informally, an image of a point source is diffraction limited (DL) if the first few Airy rings are visible. A well-accepted formal definition of DLW is that wavelength at which the Strehl ratio has value $0.8^{1}$. The Strehl ratio is the ratio of the intensity at the center of the core normalized by the corresponding value for the DL telescope ${ }^{1,2}$. With detectors that to some extent under sample the image core, a calculation of the Strehl ratio is quite challenging. The approach used here is to determine the DLW using NP as a proxy for Strehl. The IOC Spitzer Telescope NP are compared with the NP that result from a physical optics far field Fraunhofer Model ${ }^{1}$ of the prescription Spitzer Telescope and its pixel plate scale (Figure 9). In Figure 9, pixel phase is the relative location of the PSF centroid within the field of a pixel. The top curve is for the PSF centroid located at the intersection 4 pixels and has a short NP wavelength limit of 4 . When the PSF centroid is at the center of the line between 2 pixels, the short wavelength limit is 2 . NP $=1$ When the PSF centroid is located at the center of a pixel that is much larger than the PSF. There exists a the long wavelength limit beyond which imaging is independent of pixel phase. For the Spitzer telescope imaging with pixels 1.2 arcsecond on the sky, NP are independent of pixel phase for wavelengths longer than $\mu \mathrm{m}$. This wavelength demarcates the transition between overand under-sampling. Noise-pixels are calculated over a detector sub-array of 13 by 13 pixels $(n=169)$. The modeled 


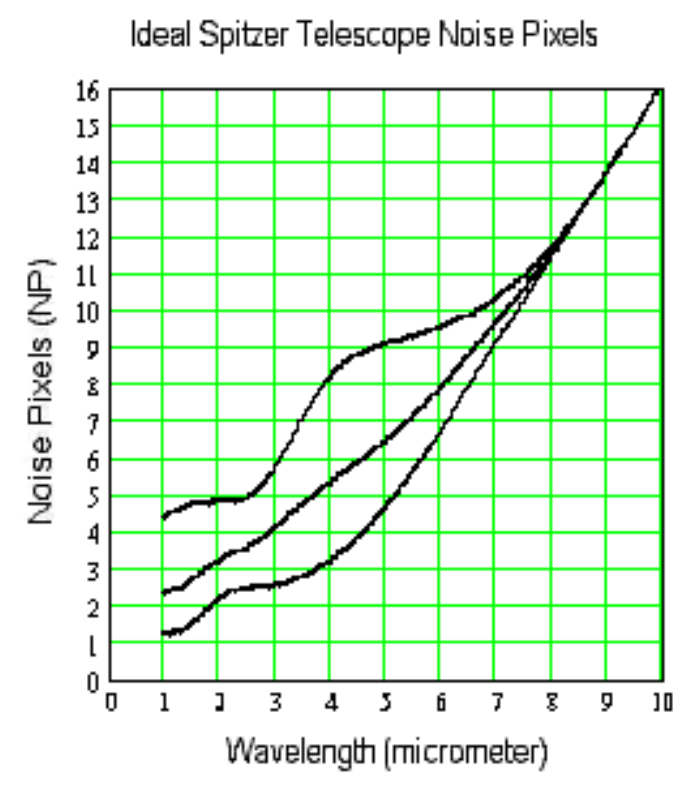

Figure 9: Plotted is the number of noise-pixels produced by Airy model of the Spitzer prescription telescope plotted against wavelength when imaging with a pixel plate scale corresponding to the IRAC instrument, with pixel phase as a parameter.

Spitzer telescope has a 0.85 -m entrance aperture, a $0.32-\mathrm{m}$ central obscuration diameter, and is imaging at the Cassegrain focus with pixels that are 1.2 arcseconds on the sky.

\subsection{Methods and results}

The plots of figure 9 are calculated by direct numerical integration by sampling the obscured Airy function ${ }^{11}$ over each of a square 169 pixel (13x13 pixels) sub-array. At a wavelength of one micrometer, one pixel ensquares almost 2 Airy rings. Over each pixel the Airy function is sampled on a 100 by 100 grid. The calculation is repeated 91 times to cover the wavelength range of 1 to 10 micrometer in 100 nanometer wavelength steps. The calculation is repeated for each of the 3 pixel phases shown. The direct numerical integration is programmed in FORTRAN using Ooura ${ }^{12}$ fast Bessel functions subroutines. Because telescope secondary mirror support spiders are not included in this calculation, the plotted NP represent a lower achievable NP limit, and the DLW determinations made by comparing actual NP to the values of this plot are conservative. In orbit, the smallest number of NP were produced by IRAC channel 1 . For this channel NP $=7.01$, according to the tabular form of figure 9 (Table 4). Thus, Spitzer is DLW at $5.4 \mu \mathrm{m}$.

\begin{tabular}{|c|c|}
\hline \multicolumn{2}{|c|}{ Spitzer In-Orbit Diffraction Limited Wavelength } \\
\hline Wavelength $(\mu \mathrm{ms})$ & Noise-pixels \\
\hline 5.40 & 7.0 \\
\hline 5.50 & 7.1 \\
\hline 5.60 & 7.3 \\
\hline
\end{tabular}

Table 4. Spitzer In-Orbit Diffraction Limited Wavelength

\section{DISCUSSION}

We can conclude from the Spitzer in orbit optical performance qualification experience that a light weight all Beryllium (Be) cryogenic telescope of 85 centimeter diameter can be figured at room temperature to compensate for an-isotropies in the coefficient of thermal expansion (CTE) of Be. These an-isotropies lead to optical figure errors at cryogenic temperatures that were corrected to meet the Spitzer LOR. The performance limit of cryogenic deformation compensation 
appears to be set not only by the quality to which the primary mirror was figured but also by gaining precise knowledge and confidence of the predicted in orbit figure through an extensive ground-based testing program. The Spitzer Project has demonstrated that it is possible to set the focus of a $10,300 \mathrm{~mm}$ focal length warm launched cryogenic telescope to within $1.7 \mathrm{~mm}$, or to one part in 6000 . The focus setting limitation is due largely to an uncertainty in the absolute calibration of the cryogenic focal length of our auto-collimation flat. The Spitzer in-orbit focus position determination and optical performance determination experience demonstrates that the focus departure and sign can be determined by minimizing the image cross correlations between IOC image data and a pre-compiled library of model images to a tolerance level comparable to the residual cryogenic figure errors of the optical model. After completing 2 in orbit focus moves we successfully predicted the residual distance to best focus by finding the minimum of the NP focus equation. Through extensive modeling analysis, the NP vs focus position curve was shown to be closely approximated by a quadratic equation. The Telescope in-orbit optical performance was determined by comparison of the NP obtained on stellar images with the NP obtained from the scalar diffraction theory model of the prescription telescope imaging in monochomatic illumination. We conclude that the in-orbit Spitzer telescope has a DLW of $5.4 \mu \mathrm{m}$. In conclusion, Spitzer meets all of its Level One Requirements with a small margin.

\section{ACKNOWLEDGMENTS}

The research described in this paper was carried out at the Jet Propulsion Laboratory, California Institute of Technology, under a contract with the National Aeronautics and Space Administration (NASA). RDG was funded for this work by NASA through the JPL SIRTF Project Office.

\section{REFERENCES}

1. M. Born and E. Wolf, Principles of Optics, $2^{\text {nd }}$ Revised Edition, p. 441, Pergamon Press, Oxford, 1964.

2. K. Strehl, Zeitschrift für Instrumkde, 22, 213, 1902.

3. W. F. Hoffmann, J. L. Hora, J. E. Mentzell, C. T. Marx, and P. R. Eisenhardt, " Simfit and Focus Diversity: methods for determining the focus of the SIRTF telescope in space without a focus slew", SPIE Proceedings 4850, pp 428-440, 2003.

4. R. D. Gehrz and E. A. Romana, "Charter and Activities of the SIRTF In-Orbit Checkout Focus Integrated Products Team and Optical Performance of the CTA”, SPIE Proceedings 4850, pp 62-71, 2003.

5. G. G. Fazio, J. L Hora, S. P. Willner, J. R. Stauffer, M. N. L. Ashby, Z. Wang, E. V. Tollestrup, J. Pipher, W. Forrest, C. McCreight, C. H. Moseley, W. F. Hoffman, P. Eisenhardt, and E. L. Wright, "The Infrared Array Camera (IRAC) for the Space Infrared Telescope Facility (SIRTF), SPIE Proceedings 3354, pp 1024-1031, 1998.

6. J. P. Schwenker, B. R. Brandl, W. F. Hoffmann, J. L. Hora, A. K. Mainzer, J. E. Mentzell, and J. E. Van Cleve, "SIRTF-CTA Optical Performance Test Results", SPIE Proceedings 4850, pp 30-41, 2003.

7. J. P. Schwenker, B. R. Brandl, W. L. Burmester, J. L. Hora, A. K. Mainzer, P. C. Quigly, and J. E. Van Cleve, "SIRTF-CTA Optical Performance Test", SPIE Proceedings 4850, pp 304-317, 20038.

8. J. R. Houck, T. L. Roellig, J. Van Cleve, B. Brandl, and K. Uchida, "IRS: the Spectrograph on the SIRTF; Its Fabrication and Testing", SPIE Proceedings 4131, pp 370-77, 2000.

9. E. T. Young, G. H. Rieke, J. Cadien, H. Dole, C. Englebracht, K. D. Gordon, G. B. Heim, D. M. Kelly, and J. A. Stansberry, "Ground test characterization of the Multiband Imaging Photometer for SIRTF (MIPS)", SPIE Proceedings 4850, pp 98-107, 2003.

10. W. F. Hoffmann, J. L. Hora, J. E. Mentzell, C. T. Marx, P. R. Eisenhardt, S. J. Carey, and S. T. Megeath, "Determination of Spitzer Space Telescope focus from IRAC images without a focus slew", SPIE Proceedings, 5487-88 (this volume), 2004.

11. D. Schroeder “Astronomical Optics”, 1999, Academic Press: New York.

12. FORTRAN double precision Bessel function subroutines: Takuya Ooura, Research Institute for Mathematical Sciences. Kyoto University, Kyoto 606-8502 Japan. Email : ooura@kurims.kyoto-u.ac.jp, http://momonga.t.u-tokyo.ac.jp/ ooura/bessel.html. 